\title{
PLANETESIMALS TO PROTOPLANETS. I. EFFECT OF FRAGMENTATION ON TERRESTRIAL PLANET FORMATION
}

\author{
Zoë M. Leinhardt and Derek C. Richardson \\ Department of Astronomy, University of Maryland, College Park, MD 20742-2421 \\ Received 2004 November 28; accepted 2005 January 31
}

\begin{abstract}
We present results from a dozen direct $N$-body simulations of terrestrial planet formation with various initial conditions. In order to increase the realism of our simulations and investigate the effect of fragmentation on protoplanetary growth, we have developed a self-consistent planetesimal collision model that includes fragmentation and accretion of debris. In our model we treat all planetesimals as gravitational aggregates so that gravity is the dominant mechanism determining the collision outcome. We compare our results to those of Kokubo \& Ida in which no fragmentation is allowed; perfect merging is the only collision outcome. After 400,000 yr of integration our results are virtually indistinguishable from those of Kokubo \& Ida. We find that the number and masses of protoplanets and the time required to grow a protoplanet depend strongly on the initial conditions of the disk and are consistent with oligarchic theory. We have found that the elasticity of the collisions, which is controlled by the normal component of the coefficient of restitution, does not significantly affect planetesimal growth over a long timescale. In addition, it appears that there is a negligible amount of debris remaining at the end of oligarchic growth, where "debris" is defined as particles too small to be resolved in our method, although we caution that these results are for an initial debris mass fraction of $1 \%$. The debris component is not massive enough to alter the dynamics of the protoplanets.
\end{abstract}

Subject headings: methods: $n$-body simulations — planetary systems - solar system: formation

Online material: color figures

\section{INTRODUCTION}

Over the past decade more than 130 Jupiter-sized extrasolar planets have been discovered. Innovations such as satellite interferometers and large ground-based surveys will allow observers to detect Earth-sized planets and increase the extrasolar planet inventory by orders of magnitude. At the same time the growing capabilities of computers make large direct simulations of solar system formation possible. Numerical simulations are essential to understanding how and under what conditions terrestrial planets form, because simulations, unlike observations, can show the evolution of a single system over a large period of time. Observations, although indispensable, can provide only instantaneous information about terrestrial planets and their environment.

Because of computational limitations, previous numerical simulations have significantly simplified planetesimal collisions, the dominant growth mechanism in the protoplanetary disk. Past simulations of terrestrial planet formation have either assumed that two colliding planetesimals merge completely (perfect merging), thus ignoring any erosion of the planetesimals, or have extrapolated the collision outcome over many orders of magnitude from a model based on laboratory impact experiments in which self-gravity is unimportant. In a real disk a range of collision circumstances are expected, from slow collisions in which most of the mass of the two colliding planetesimals ends up in the largest postcollision remnant, to fast collisions in which most of the mass ends up in small fragments. For planetesimals large enough not to be affected by nebular gas $(R>10 \mathrm{~km})$, the most important force involved in collisions is gravity. At these sizes the material strength of the planetesimals is negligible compared to their gravitational binding energy (Holsapple 1994; Asphaug et al. 2002). The first simplification method, perfect merging, ignores the range of collision possibilities. The second simplification method, extrapolation of laboratory experiments, ignores the effect of gravity in the collision outcome. In both cases the numerical simulations produce terrestrial planet systems with eccentricities many times those of our own solar system, suggesting that an important mechanism is missing (Agnor \& Ward 2002; Kokubo \& Ida 2002). More detailed modeling of the collisions between planetesimals is the next step toward making our numerical models of planet formation more realistic and complete.

We have developed the most realistic planetesimal collision model to date, in which gravity is the dominant mechanism in determining the collision outcome, and have incorporated it into a planet formation model. We have completed a series of highresolution direct numerical simulations of terrestrial planet formation. We have found that fragmentation has little effect on the growth of protoplanets after several protoplanets have formed nor is there a sufficiently massive debris component remaining to affect the dynamics of the protoplanets. This suggests that either a different eccentricity damping mechanism is required or more simulations are needed to quantify the range of possible outcomes as a function of the initial conditions.

\subsection{Previous Work on Planet Formation}

Modern theories of terrestrial planet formation are divided into four stages (e.g., Lissauer 1993): (1) the initial stage, in which dust condenses out of the hot gaseous disk surrounding the young star-significant growth of the grains is hindered by turbulence; (2) the early stage, in which dust grains grow from centimetersized particles to kilometer-sized planetesimals by accretion - gas drag circularizes the orbits; (3) the middle stage, in which planetesimals grow into protoplanets, again by accretion, but gravitational forces dominate - dynamical friction and the redistribution of energy via collisions causes large objects to maintain nearly circular orbits (low eccentricity and inclination) while the smaller bodies become excited (high eccentricity and inclination); and 
(4) the late stage, in which runaway accretion terminates due to lack of smaller material within the feeding zone of the protoplanetsthe protoplanets grow into planets via long-term, long-distance, cumulative gravitational interactions. The initial and early stages of planet formation have proven the most difficult to model in a detailed way because of complex, uncertain physics. The early stage of planet formation ends when the masses of the largest planetesimals significantly exceed the mass in gas that they intercept over one orbit; for planetesimal internal density $\rho \sim 2 \mathrm{~g} \mathrm{~cm}^{-3}$ and gas density $\rho_{g} \sim 2 \times 10^{-9} \mathrm{~g} \mathrm{~cm}^{-3}$ at $1 \mathrm{AU}$, this occurs at planetesimal sizes of $1-10 \mathrm{~km}$ in radius. The middle and late stages are much more straightforward to model directly since the planetesimals are large enough that gravity is the dominant force. Thus, most of the numerical work on planet formation has focused on these later phases of planet formation.

There are two complementary quantitative approaches that have been used to investigate the middle and late stages of planet formation: statistical methods (Greenberg et al. 1978; Wetherill \& Stewart 1989, 1993) and direct numerical methods (Lecar \& Aarseth 1986; Beaugé \& Aarseth 1990; Kokubo \& Ida 1996, 1998, 2000, 2002; Richardson et al. 2000). The statistical method treats planetesimals as analogs to gas molecules and applies a method similar to the kinetic theory of gases to treat the evolution of planetesimals (Safronov 1969; Greenberg et al. 1978). Statistical methods are very powerful at the beginning of the middle stage of planet formation when the number of planetesimals is large and the planetesimal population can be accurately described as a thermal distribution. In addition, the statistical method can take into account any effect that can be described analytically such as gas drag, dynamical friction, and fragmentation. Using this method, Wetherill \& Stewart (1989) found that planetesimals go through a runaway growth phase in which the largest planetesimals grow faster than any other planetesimal because of the equipartition of energy from dynamical friction. This causes the larger planetesimals to separate from the background population of smaller planetesimals. At this point the gas dynamics treatment of the planetesimal population begins to break down because the spatial distribution is no longer homogeneous ( Wetherill \& Stewart 1993).

Direct numerical simulations can be integrated through the runaway growth phase and are limited only by computer capabilities, but they are much more computationally expensive. The largest direct simulation published of planet formation integrated through runaway growth uses $10^{4}$ particles (Kokubo \& Ida 2002). These direct simulations show two phases of planetesimal growth: first, runaway growth, and second, oligarchic growth of protoplanets (planet embryos), in which large protoplanets grow more slowly than smaller protoplanets but all protoplanets continue growing faster than the background planetesimals. Kokubo \& Ida (2002) simplify planetesimal collisions by neglecting erosion, thereby assuming that planetesimal collisions always result in growth. This simplification may have a complex effect on the timescale of planet formation and the final outcome because the balance between growth and erosion of planetesimals is ignored. Other numerical simulations (Beaugé \& Aarseth 1990) took into account fragmentation of planetesimals (in a very low resolution two-dimensional $N$-body simulation) using a semianalytical prescription similar to that employed in statistical simulations (Wetherill \& Stewart 1993). The effects of impact angle, spin, and the mass ratio of the colliding bodies are not taken into account in either prescription. In order to insure that our simulations correctly include as many effects of planetesimal collisions as possible, we model them directly or interpolate from a table of our previous impact simulations.
The remainder of our paper is divided into four parts: $\S 2$ presents our numerical method in detail, $\S 3$ discusses our results in the context of previous numerical simulations, $\S 4$ summarizes our findings, and $\S 5$ suggests future work.

\section{NUMERICAL METHOD}

We use the highly efficient $N$-body gravity code PKDGRAV for our simulations, which has been modified to resolve collisions realistically and account for the accretion of dust onto planetesimals. In this section we describe the numerical methods we use for the planetesimals, the planetesimal collisions, the unresolved debris, the planetesimal disk, and the integration.

\subsection{Planetesimal Structure Model}

There is significant observational evidence that small bodiesasteroids and comets - in our solar system are gravitational aggregates or "rubble piles" (objects with little or no tensile strength held together by gravity), not coherent objects (see Leinhardt et al. 2000; Richardson et al. 2002). For example, several asteroids have giant craters and low bulk densities, and almost all are rotating slower than the rubble-pile breakup limit; of the 984 observed, none with diameters larger than $150 \mathrm{~m}$ are spinning faster than this limit (Pravec et al. 2002). The evidence suggests many asteroids are likely made of loosely consolidated material and therefore contain a large fraction of void space. The voids impede the transmission of energy from collisional shocks and allow a rather weak body to survive what would otherwise be a catastrophic impact event (Ryan et al. 1991; Love \& Ahrens 1996; Asphaug et al. 1998). However, it is unclear whether asteroids are a fair representation of planetesimals since asteroids have been collisionally processed during their lifetime. Nonetheless, even if planetesimals were originally coherent, the strength due to self-gravity of the planetesimal is many orders of magnitude larger than the material strength (Holsapple 1994).

Observations of comets suggest that they are also gravitational aggregates. The most impressive example of this was the tidal disruption of comet D/Shoemaker-Levy 9 (SL9) by Jupiter in 1993. The disruption showed that SL9 was fragile, with little or no tensile strength (Asphaug \& Benz 1996). Comets are much more pristine than asteroids and have not been as significantly altered by collisions as main-belt asteroids.

Thus, in light of the observational evidence that a large percentage of small bodies in our solar system may be gravitational aggregates, and the understanding that planetesimals are large enough that their gravitational strength is significantly larger than their material strength, we have chosen to model planetesimals involved in collisions in the nebular disk as "perfect" rubble piles (Richardson et al. 2005).

\subsection{Planetesimal Collision Model}

The growth of planetesimals into protoplanets is dominated by planetesimal-planetesimal collisions. The solar system formation simulations presented here use a two-phase process to determine the collision outcome. In the first phase the collision parametersrelative speed, impact parameter, and mass ratio of the projectile to the target ( $v, b$, and $\mu$, respectively) - are used to interpolate/ extrapolate the mass of the largest postcollision remnant from a collision outcome database. Spin of individual planetesimals is not a parameter in the database because the number of possible target-projectile spin vector orientations is large and thus difficult to parameterize. In addition, the direction of the spin vectors of the planetesimals should be randomized. Therefore, on average 


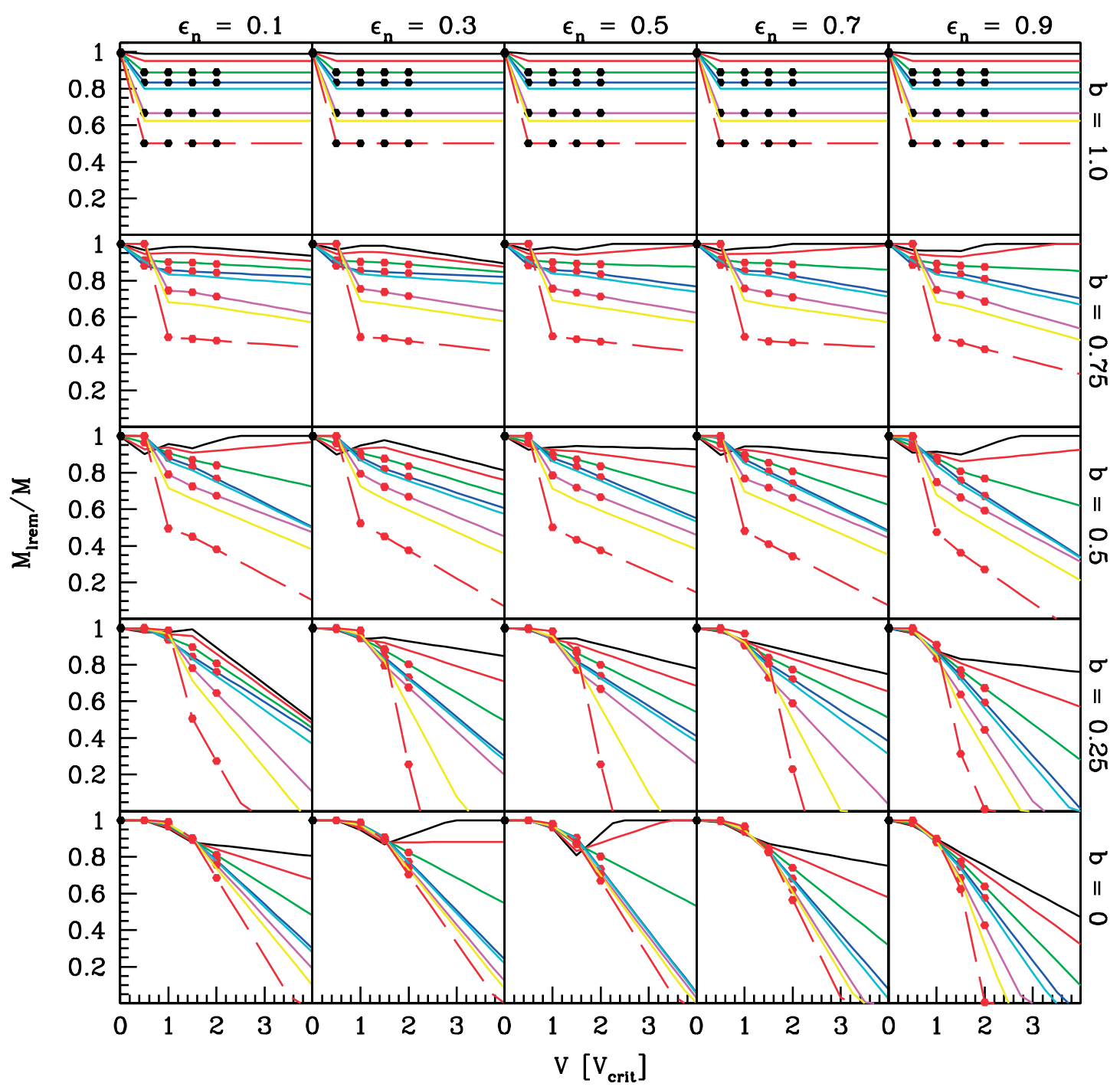

FIG. 1.- Interpolation/extrapolation table for the first phase of the collision model. Each plot in this table shows the mass of the largest postcollision remnant in units of the total system mass vs. impact speed in units of $v_{\text {crit }}$ (see text). The five columns correspond to different normal coefficients of restitution $\left(\epsilon_{n}\right)$. No surface friction was included in any of these simulations $\left(\epsilon_{t} \equiv 1\right)$. The rows correspond to different impact parameters $b$ in units of the sum of the radii of the impactors ( $b=0$ is a head-on collision, $b=1$ is a glancing collision). The color lines represent various mass ratios $(\mu)$ : black, 1/100; red, 1/20; green, 1/9; blue, 1/6; cyan, 1/5; magenta, 1/3; yellow, 1/2; red dashes, 1/1. The red dots are actual data from numerical simulations (similar to the one shown in Fig. 3 ). The black dots are points in the database that are fixed at theoretical limits.

the spin of the planetesimals should not affect the first-order approximation of the collision outcome (see Leinhardt et al. 2000; Leinhardt \& Richardson 2002 for discussion of the effect of spin on collision outcome). The collision database consists of the results of several hundred rubble-pile planetesimal collisions over a wide range of parameter space (an extension of Leinhardt et al. 2000; Leinhardt \& Richardson 2002).

Figure 1 shows the mass of the largest postcollision remnant, $M_{\text {lrem }}$, in units of system mass $(M$, the sum of the projectile and target mass, $M_{\text {proj }}+M_{\text {targ }}$ ) versus impact speed. Figure 2 shows the same results with $M_{\text {lrem }}$ in units of $M_{\text {targ }}$. The columns represent different normal coefficients of restitution $\left(\boldsymbol{v}^{\prime}=-\epsilon_{n} \boldsymbol{v}_{n}+\epsilon_{t} \boldsymbol{v}_{t}\right.$, where the impact velocity $\boldsymbol{v}=\boldsymbol{v}_{n}+\boldsymbol{v}_{t}, \boldsymbol{v}_{n}$ is the component of the impact velocity normal to the plane of impact, $\boldsymbol{v}_{t}$ is the component tangent to the impact plane, and $\boldsymbol{v}^{\prime}$ is the postimpact velocity). The rows represent various impact parameters in units of the sum of the projectile and target radii, $R_{\text {proj }}+R_{\text {targ }}$. The red points on these figures are results from actual simulations (see Fig. 3 for an example). The black points are theoretical limits: $M_{\text {lrem }}$ is fixed at 1 for $v=0$ and at the mass of the target for $b=1$. The colored lines are interpolation or extrapolation from these data points.

In order to increase the flexibility of the database, the impact speed in the database is in units of

$$
v_{\text {crit }} \equiv M \sqrt{\frac{6 G}{5 \mu_{r} R_{V}}}
$$

where $R_{V} \equiv\left(R_{\text {proj }}^{3}+R_{\text {targ }}^{3}\right)^{1 / 3}$ is the radius of a spherical body with the combined volume of the projectile and target, assuming equal bulk density, and $\mu_{r} \equiv M_{\text {proj }} M_{\text {targ }} / M$ is the reduced mass. Here $v_{\text {crit }}$ is found by equating the total kinetic energy to the gravitational binding energy $\left(v_{\text {crit }}=1\right.$ is the approximate speed necessary for catastrophic dispersal when the largest remnant is $50 \%$ of the original system mass; see Leinhardt et al. 2000). This means that when a collision is predicted the impact speed is converted into $v_{\text {crit }}$ units that scale with binding energy, allowing the same database to be used for planetesimals that 


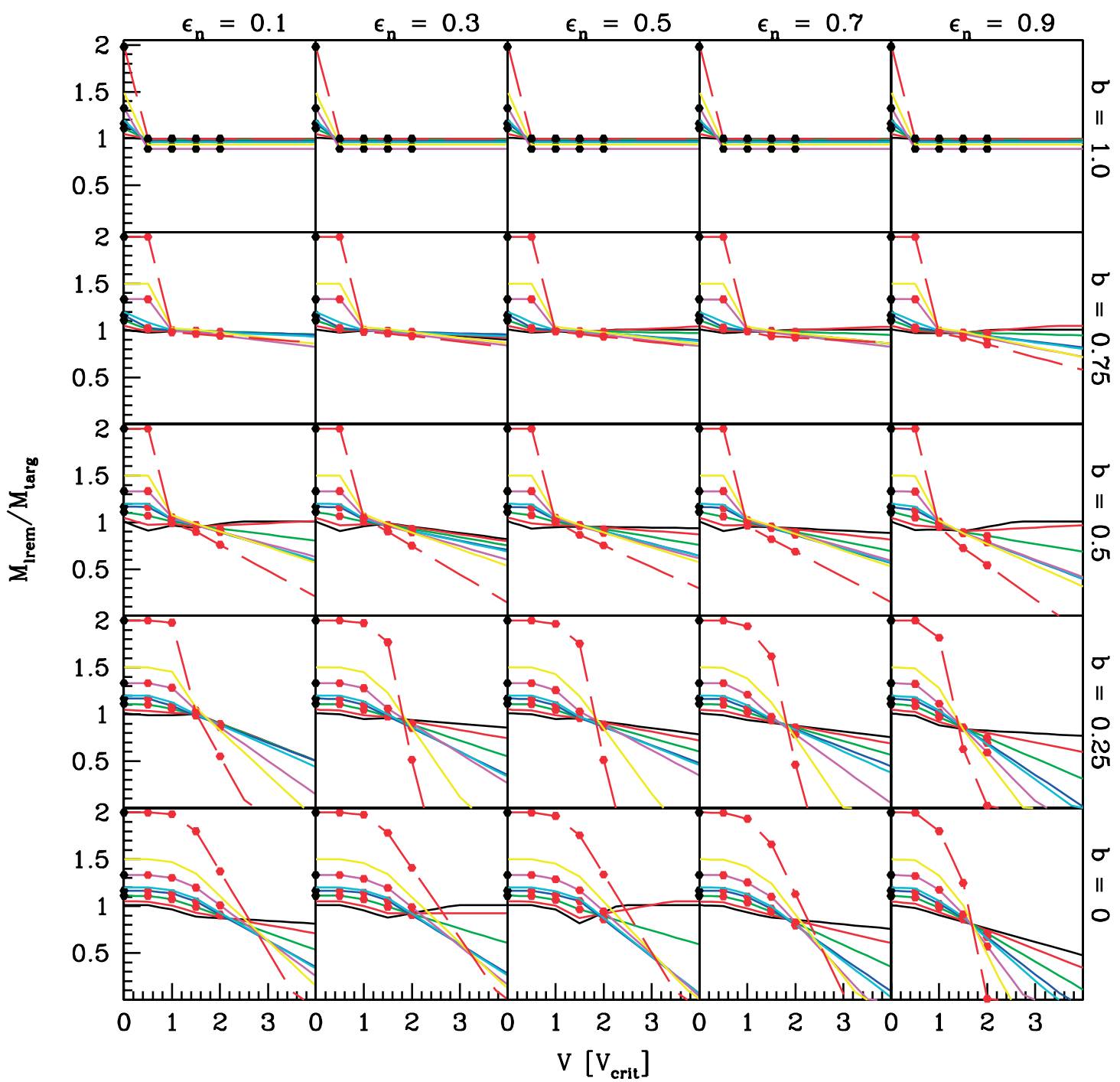

Fig. 2.-Same as Fig. 1, but the mass of the largest postcollision remnant is measured with respect to the initial mass of the target, separating the lines of different mass ratio at low impact speed.

have bulk densities different from those used to create the database; $v_{\text {crit }}$ is proportional to the mutual escape speed $v_{\text {esc }} \equiv$ $(2 G M / R)^{1 / 2}$, except for cases of extreme mass ratio. Figure 4 shows how the ratio

$$
\frac{v_{\text {crit }}}{v_{\text {esc }}}=(1+\mu) \sqrt{\frac{3}{5 \mu} \frac{1+\mu^{1 / 3}}{(1+\mu)^{1 / 3}}}
$$

varies with $\mu$, the mass ratio of the projectile to the target, and the mutual escape speed.
Each planetesimal used in the database was made up of a fixed number of identical self-gravitating hard spheres (Fig. 3). Inelastic bouncing was the only possible collision outcome between the spheres: no mergers or fragmentation of particles were allowed. All simulations used a direct numerical method ( $(2.5)$ to evolve the positions and velocities of the rubble-pile particles under the constraints of gravity and physical collisions.

If the collision outcome from the database is one large body with a small amount of debris, this outcome is used in the simulations as the result of the planetesimal collision. In other words, the colliders are replaced with the largest postcollision remnant

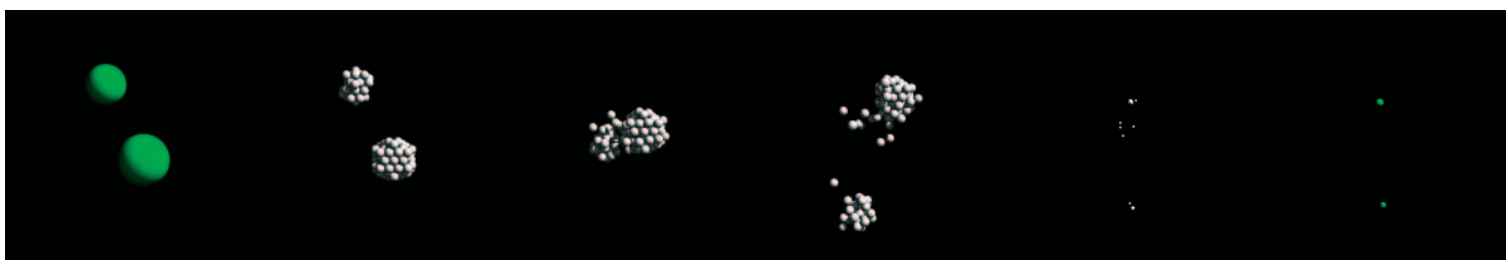

Fig. 3. - Snapshots of a collision, with time increasing to the right. The two planetesimals have a mass ratio of $\frac{1}{2}$. The impact parameter is $b=0.89$, and the initial relative speed is $1.5 \mathrm{~m} \mathrm{~s}^{-1}$. The initial rubble piles consist of a large number of hard spheres held together by their mutual gravity. Individual spheres are indestructible and bounce off one another inelastically. 


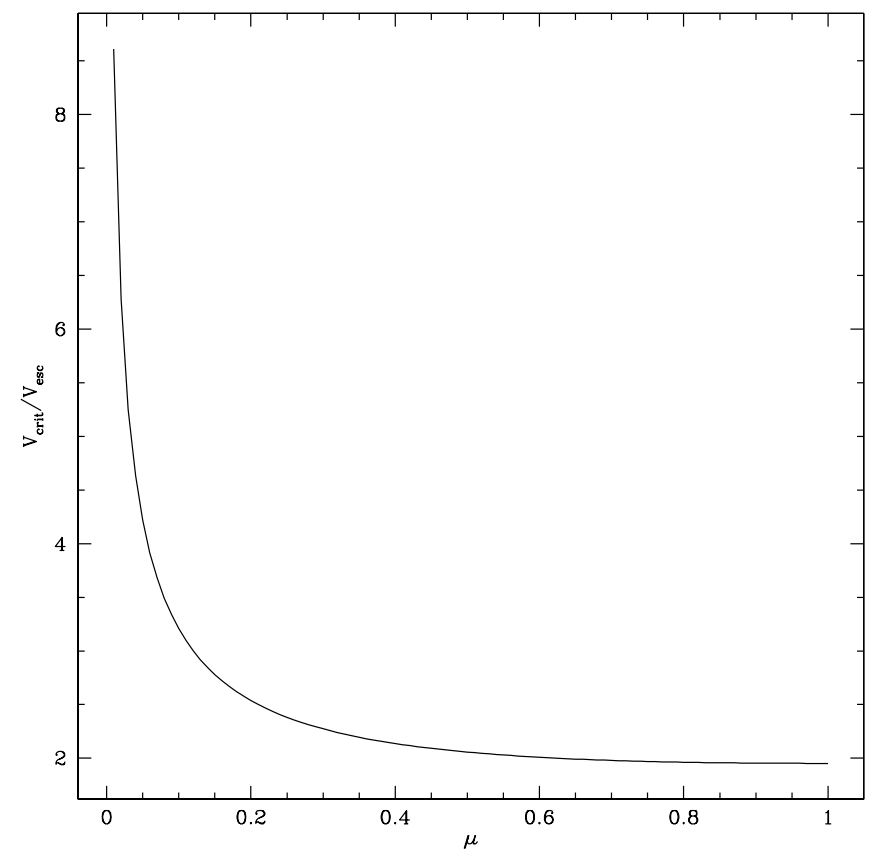

FIG. 4.- Ratio of $v_{\text {crit }}$ to $v_{\text {esc }}$ as a function of the mass ratio $\left(\mu=M_{\text {proj }} / M_{\text {targ }}\right)$, assuming equal mass density.

from the database. The rest of the mass from the original planetesimals is considered unresolved debris and is tracked in a semianalytic way by the numerical code $(\S 2.3)$.

If the collision outcome predicted by the database consists of two or more massive remnants, the planetesimals involved in the collision, which were modeled as single particles up to this point, are substituted by actual rubble piles and the collision is then integrated directly. The total mass, bulk density, and angular momentum of the original planetesimals are preserved. The solar system formation simulation proceeds as before except for the inclusion of the rubble-pile planetesimals (see $\S 2.5$ for rubblepile time step). The number of particles in each rubble pile is between 100 and 2500, depending on the size of the target. Each particle in the rubble pile is constrained to be smaller than the initial size of the planetesimals at the beginning of the simulation (the resolution limit of the simulation; $\S 2.3$ ). Initially, a rubble pile is created with 100 particles. If the particles in the rubble pile are larger than the resolution limit, the number of particles is increased.

For 10 dynamical times $\left(\tau_{\text {dyn }} \sim 1 / \sqrt{G \rho}\right.$, where $G$ is the gravitational constant and $\rho$ is the bulk density of the planetesimal), rubble-pile particles bounce when they collide with each other, allowing the collision remnants to reach equilibrium (many remnants will be gravitational aggregates; see Leinhardt et al. 2000; Michel et al. 2001). After 10 dynamical times the rubble-pile particles merge with each other. This means that any gravitationally reaccreted remnants become single particles at this point in the simulation. After 20 dynamical times any remaining collisional debris that is smaller than the resolution limit is demoted to "unresolved debris" and is no longer followed directly; the mass is incorporated into the unresolved debris component.

\subsection{Unresolved Debris}

In order to handle debris either created by planetesimal collisions or existing initially as part of the starting conditions, we divide the planetesimal disk into a configurable number of cylindrical annuli. Any particles smaller than the resolution limit (usually taken as the radius of the starting planetesimals) are binned in the annulus at that radius. The debris particles are assumed to be on planar circular orbits. The larger planetesimals sweep up the debris as they pass through the annuli, thereby growing in mass, according to

$$
M_{p}^{\prime}=M_{p}+\delta m
$$

where $M_{p}$ is the original mass and $\delta m$ is the mass accreted given by

$$
\delta m=e \pi R^{2} 2 \pi a \rho \frac{\delta t}{P}
$$

where $e$ is the planetesimal's eccentricity, $R$ is its physical radius, $a$ is the semimajor axis of its orbit, $\rho$ is its mass density, $\delta t$ is the time since the last dust accretion update, and $P$ is the Keplerian period corresponding to $a$. The accretion of the debris causes the orbits of the larger planetesimals to circularize; the accretion of the dust by the planetesimal is assumed to conserve linear momentum, and thus the velocity components are updated according to

$$
\begin{aligned}
& v_{x}^{\prime}=v_{k x}+\frac{M_{p}}{M_{p}^{\prime}}\left(v_{x}-v_{k x}\right), \\
& v_{y}^{\prime}=v_{k y}+\frac{M_{p}}{M_{p}^{\prime}}\left(v_{y}-v_{k y}\right), \\
& v_{z}^{\prime}=\frac{M_{p}}{M_{p}^{\prime}} v_{z},
\end{aligned}
$$

where $\boldsymbol{v} \equiv\left(v_{x}, v_{y}, v_{z}\right)$ is the initial velocity of the planetesimal, $\boldsymbol{v}^{\prime} \equiv\left(v_{x}^{\prime}, v_{y}^{\prime}, v_{z}^{\prime}\right)$ is the updated velocity, and $\boldsymbol{v}_{k}$ is the instantaneous Kepler velocity at the planetesimal's location.

The planetesimals' mass and velocity components are updated several times per orbit. The mass accreted by a planetesimal in each update is equal to the product of the mass density of debris in the annulus, the cross-sectional area of the planetesimal, and the fraction of the orbit the planetesimal has traveled since the last update (eq. [4]).

\subsection{Planetesimal Disk Model}

In this paper we present two sets of simulations. The first set contains nine high-resolution $\left(N=10^{4}\right)$ simulations of various initial disk masses and surface density distributions to investigate the effect of fragmentation and environment on protoplanet formation (see $\S 3$ ). The standard model for a planetesimal disk assumes a "minimum-mass solar nebula" $\left(M_{\text {solid }}=0.01 M_{\odot}\right)$, a surface density at $1 \mathrm{AU}$ of $\Sigma_{1} \sim 10 \mathrm{~g} \mathrm{~cm}^{-2}$, and a surface density distribution of solid material $\Sigma_{\text {solid }}=\Sigma_{1}(a / 1 \mathrm{AU})^{-\alpha}$, with $\alpha=$ 1.5. We also simulated disks that are more and less massive than the standard model $\left(\Sigma_{1}=100,1 \mathrm{~g} \mathrm{~cm}^{-2}\right)$, as well as disks in which the mass is distributed more and less steeply $(\alpha=2.5,0.5)$. Each of these simulations begins with a 1 AU wide band of particles centered at $1 \mathrm{AU}$. The simulations are run for at least $5 \times 10^{5} \mathrm{yr}$-long enough to get through the runaway growth phase and show the formation of multiple protoplanets. The initial conditions chosen for these simulations are similar to those used by Kokubo \& Ida (2002). This allows us to compare our results to theirs and thus understand how different collision outcomes affect the formation of planets in various environments.

The second set of simulations presented in this paper consist of three lower resolution runs $(N=4000)$, each employing a different coefficient of restitution to investigate the effect of elasticity 
on planetesimal growth $(\S 3.4)$. These simulations begin with a $0.085 \mathrm{AU}$ band of equal-sized planetesimals at $1 \mathrm{AU}$ and a standard model surface density distribution with $\Sigma_{1}=10 \mathrm{~g} \mathrm{~cm}^{-2}$ and $\alpha=1.5$.

In all of these simulations the planetesimal collision model described in $\S 2.2$ is used. All planetesimals have an initial bulk density of $2 \mathrm{~g} \mathrm{~cm}^{-3}$. Like Kokubo \& Ida (2002) we are forced to employ a radial expansion parameter in order to complete our simulations in a reasonable amount of time. In order to stay consistent with previous work, we chose an expansion parameter of $f=6$ for all simulations (see Kokubo \& Ida 2002 for a discussion of the numerical effects of using $f>1$ ). ${ }^{1}$ As a result of the expansion parameter, all planetesimals actually have a bulk density of $0.00925 \mathrm{~g} \mathrm{~cm}^{-3}$. Initially the planetesimals are given random velocities with respect to the Keplerian velocity in directions both in and out of the plane chosen from a Rayleigh distribution. The peak of the distribution is set by the escape speed from the largest starting planetesimal. The exact starting velocity distribution is not critical since the relaxation timescale of the planetesimal disk is short $\left(\sim 10^{3} \mathrm{yr}\right)$ compared to the length of the simulation (Kokubo \& Ida 1996). Each simulation presented here was run on our local computer cluster. ${ }^{2}$ Each high-resolution simulation took about 1 month to complete, while the lower resolution simulations each took about 1 week on single processors.

\subsection{Numerical Algorithm}

Our numerical simulations use a modified version of PKDGRAV (Stadel 2001; Richardson et al. 2000), a parallelized, hierarchicaltree $N$-body code that calculates gravity in $O(N \log N)$ time. The code has been modified to include the planetesimal collision model ( $\S 2.2$ ) by adding a module that uses the collision outcome database to determine whether a fully resolved collision is required. If a resolved collision is necessary, this module is responsible for substituting single-particle planetesimals with rubble piles before the collision and substituting rubble piles with single particles and unresolved debris after the planetesimal collision is complete.

The equations of motion in our simulations are integrated using a second-order leapfrog integrator with multistepping. ${ }^{3} \mathrm{Col}-$ lisions are predicted at the beginning of each position (drift) step by keeping the particle velocities fixed and extrapolating the particle positions. Once the collision outcome has been determined and new velocities (kicks) have been calculated, the postcollision particles are traced back to the start of the drift step so that they can be included in any remaining collision checks. This ensures that all collisions are detected and treated in the correct order, even if particles are involved in more than one collision during the drift step.

Since the dynamical time of a rubble pile (hours) and the orbital time of the planetesimal around the Sun $(\sim 1 \mathrm{yr})$ differ by orders of magnitude, we use a two-phase time step to increase the efficiency of our simulations. Initially all planetesimals are on the major time step $(0.01 \mathrm{yr})$. Once a collision is predicted, the time

\footnotetext{
1 Because of the expansion factor we do not test for excessive spin; the low density would force almost all interpolated collision outcomes to be resolved. As a result, we can say nothing about the spin of the protoplanets in the simulations presented here.

2 The "borg" is owned and operated by the Center for Theory and Computation (http://www.astro.umd.edu/ctc) in the Department of Astronomy at the University of Maryland, College Park.

3 For the two-body problem, without multistepping or collisions PKDGRAV is symplectic: for a planetesimal at $1 \mathrm{AU}$, eccentricity of 0.01 , and time step of $0.01 \mathrm{yr}$, the energy error is bounded and never exceeds $2 \times 10^{-3} \%$ during an orbit and never increases in time; for the same time step at $0.5 \mathrm{AU}$, the energy error is $\leq 2 \times 10^{-2} \%$.
}

step of the two planetesimals involved is reduced by a factor of 64. This means that gravity is calculated 64 times for the colliding particles, while gravity is calculated once for the rest of the particles. All particles are drifted consistently through the major step, but the colliding particles also have their kicks recalculated on the minor steps. In addition, the radius of the planetesimals is increased by a factor of 2.5 during the collision search to reduce the number of missed collisions and increase the accuracy of close approaches.

\section{RESULTS}

\subsection{Comparison with Kokubo \& Ida (2002)}

In this section we present a direct comparison of our global simulations of protoplanetary growth for different initial environments with that of Kokubo \& Ida (2002). They used a simple perfect merging prescription to determine the collision outcome from planetesimal collisions. In order to determine the effect of our gravity-dominated collision model, we have completed a series of simulations similar to theirs. We begin the comparison of our results with the standard model.

\subsubsection{The Standard Model}

In our global standard model we integrated 10,000 equalsized planetesimals for 500,000 yr. Recall that the planetesimals were placed between 0.5 and $1.5 \mathrm{AU}$ with $\Sigma=\Sigma_{1}(a / 1 \mathrm{AU})^{-\alpha}$, where $\Sigma_{1}=10 \mathrm{~g} \mathrm{~cm}^{-2}$ and $\alpha=3 / 2$. Figure 5 shows the location of the planetesimals and protoplanets on the semimajor axiseccentricity and semimajor axis-mass planes at four times during the simulation. The filled circles in Figure $5 a$ represent those planetesimals that have grown larger than 100 times their initial mass (these are the protoplanets). The error bars are 10 Hill radii $\left(r_{\mathrm{H}}\right)$ wide, the approximate separation expected due to orbital repulsion (Kokubo \& Ida 1995), where

$$
r_{\mathrm{H}} \equiv\left(\frac{2 M}{3 M_{*}}\right)^{1 / 3} a
$$

$M$ is the mass of the protoplanet, $M_{*}$ is the mass of the central star (always $1 M_{\odot}$ ), and $a$ is the semimajor axis of the protoplanet.

In each stage of the simulation shown in Figure 5 we found roughly the same number of protoplanets as Kokubo \& Ida (2002), but we had about one-half to two-thirds as many planetesimals. By $400,000 \mathrm{yr}$ (Fig. 5, bottom panels) we had 12 protoplanets and 236 planetesimals, which is similar to the Kokubo \& Ida (2002) result of 12 protoplanets and 333 planetesimals. The protoplanets have relatively low eccentricity because of the dynamical friction from the planetesimals. The largest protoplanet is $\sim 1500$ times the initial planetesimal mass after 400,000 yr. Figure $5 b$ shows that the 12 protoplanets that have grown by this time are separated by at least 2 orders of magnitude in mass from the background planetesimal population. Note that all of the times that are used here apply to the "real" time growth of the artificially expanded planetesimals $(f=6)$. The growth timescale for uninflated planetesimals varies as $1 / f^{2}$ until gravitational focusing becomes effective, at which point the growth timescale varies as $1 / f$ (Kokubo \& Ida 1996).

Figure 6 shows the eccentricity of all particles in the simulation at four times during the simulation as a function of mass. By 400,000 yr the protoplanets have low eccentricity and have begun to stir up the eccentricities of the small planetesimals to $e>0.1$ (Figs. 5 and 6 , bottom panels) via viscous stirring. The highest eccentricity of the planetesimals is $\sim 0.27$, about 3 times 

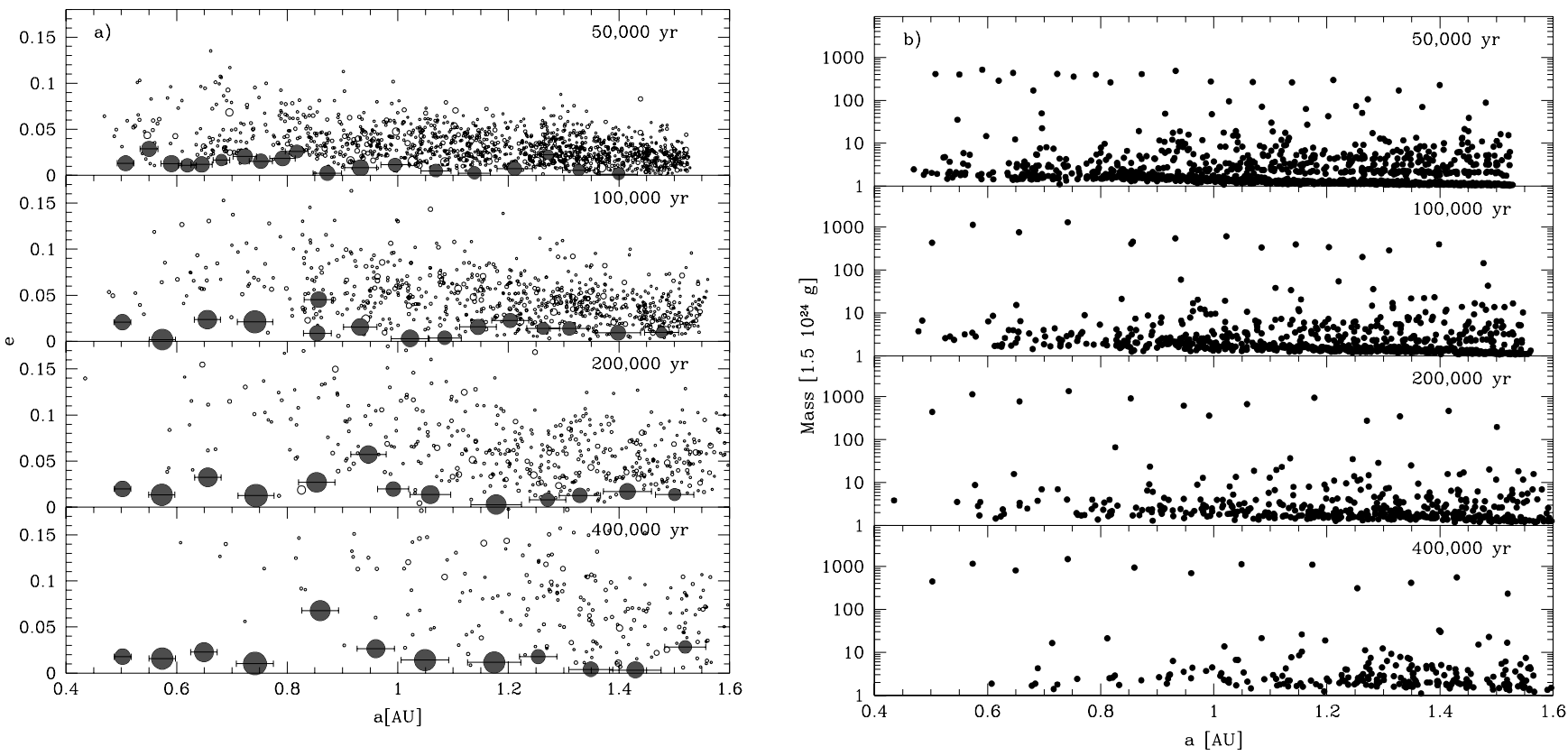

FIG. 5.- (a) Semimajor axis vs. eccentricity for all particles in the standard model after 50,000, 100,000, 200,000, and 400,000 yr. The radius of each circle is proportional to the radius of the particles in the simulation. The filled circles represent those protoplanets that have reached masses greater than 100 times the starting planetesimal mass $\left(1.5 \times 10^{24} \mathrm{~g}\right)$. The horizontal error bars are $10 r_{\mathrm{H}}$ in length. $(b)$ Same as $(a)$, but for semimajor axis vs. mass in units of starting mass. [See the electronic edition of the Journal for a color version of this figure.]

the "escape eccentricity" from the largest protoplanet (the escape speed divided by the Keplerian speed at the semimajor axis of the protoplanet; see $\S 4.3$ and eq. [21] of Kokubo \& Ida 2002). Both the escape eccentricity and the largest eccentricity of the planetesimals are consistent with the values found by Kokubo \& Ida (2002).

Figure 7 shows the cumulative number of particles in a given mass bin at five stages of evolution in the simulation. The planetesimal disk is also divided into four radial bins in this figure.

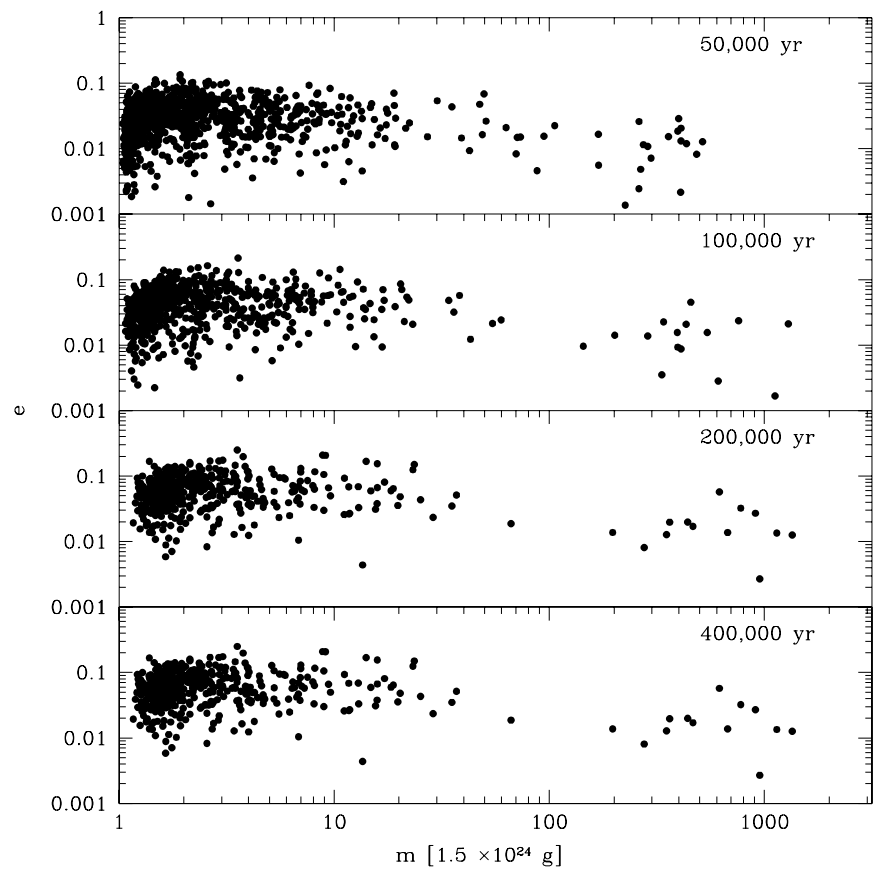

FIG. 6.-Shows the positions of all particles in the standard model simulation in mass vs. eccentricity space at four different times during the simulation. The mass is in units of the initial mass.
Comparison of this plot with that of Kokubo \& Ida (2002, their Fig. 4) reveals that our simulations initially evolve more quickly than theirs. By 50,000 yr all regions of our disk are flattening in mass distribution. Kokubo \& Ida (2002) still have quite steep distributions in the outer regions of the planetesimal disk at this point. In addition, the most massive protoplanet in the outermost radial bin is just under 200 times the initial mass at 50,000 yr;

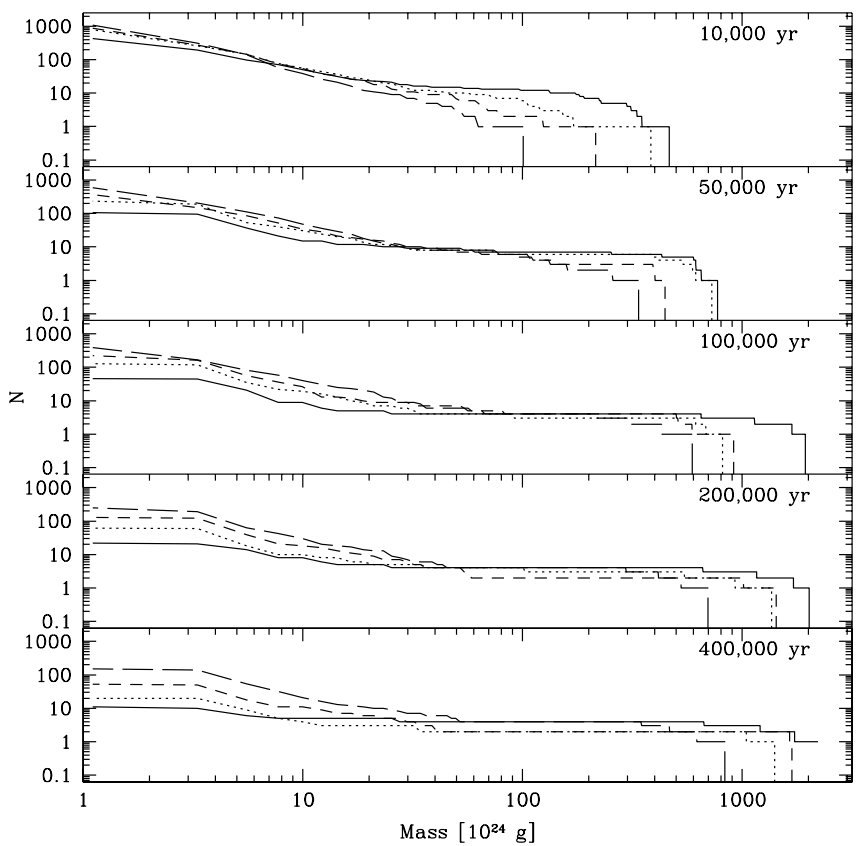

Fig. 7. - Cumulative number of particles by mass bin for five different stages in the simulation. Each line represents a different radial bin of the disk: the solid line represents the innermost region of the disk $(a<0.75 \mathrm{AU})$, the dotted line represents particles between 0.75 and $1.00 \mathrm{AU}$, the short-dashed line represents particles between 1.00 and $1.25 \mathrm{AU}$, and the long-dashed line represents particles with $a>1.25 \mathrm{AU}$ 
Kokubo \& Ida's most massive protoplanet at this time is just 50 times the initial mass. However, by $400,000 \mathrm{yr}$ the simulations appear virtually identical. Both show flattening of the mass distribution in all radial bins, with the most massive in each radial bin clustering around 1000 times the initial mass.

Although the initial evolution is faster than that seen in Kokubo \& Ida (2002), the nature of the evolution is similar. Namely, the slope of the mass distribution early in the simulations (shown in the top panel of Fig. 7) is characteristic of runaway growth (Kokubo \& Ida 2000; Makino et al. 1998), $d \log n_{c} / d \log m \simeq$ -1.5 , where $n_{c}$ is the cumulative number of planetesimals and $m$ is the mass of the planetesimals in units of $10^{24} \mathrm{~g}$. As time increases the slope becomes less steep as the number of small planetesimals drops. In Kokubo \& Ida (2002) there was no source of small planetesimals to replenish the low mass end of the mass distribution. Our collision model allows for a resupply of small planetesimals via fragmentation events. However, the resupply of small planetesimals is not significant and we observe behavior similar to that seen by Kokubo \& Ida (2002) in the reduction of the steep mass distribution slope as runaway growth transitions into oligarchic growth.

There are several reasons that could explain why our simulation initially evolved more quickly than that of Kokubo \& Ida (2002): (1) the simulations are stochastic in nature: the initial conditions are randomized, resulting in a significant diversity of outcomes (see § 3.4); (2) both our numerical integrator and our collision detection technique are quite different than those used by Kokubo \& Ida (2002) - we use a second-order integrator and small time steps to handle close approaches and collisions, whereas Kokubo \& Ida (2002) use a Hermite integrator with hierarchical time steps; (3) our model includes fragmentation. We have tested the resolution of our time steps by running the same initial condition with time steps 4 times smaller. The initial evolution is consistent with the results presented here. In addition, we have investigated the effect of a coefficient of restitution on the growth and evolution of protoplanets ( $\S 3.4)$. We see no obvious trend with the coefficient of restitution and the mass of the most massive object. However, it is possible that the collision model does affect the early stages of planetesimal growth.

\subsubsection{Surface Density Simulations}

We have investigated the effect of varying surface density by integrating three different surface density distributions $\left(\Sigma_{1}=1\right.$, 10 , and 100) for 500,000 yr. For the simulations presented in this section, $\alpha=3 / 2$. All three simulations started with 10,000 planetesimals distributed between 0.5 and $1.5 \mathrm{AU}$. The initial mass of the planetesimals was $1.5 \times 10^{23}, 1.5 \times 10^{24}$, and $1.5 \times 10^{25} \mathrm{~g}$, respectively. Figure 8 shows the results of the simulations in semimajor axis versus eccentricity space. The filled circles represent the protoplanets that have grown larger than 100 times the initial mass of the planetesimals. The horizontal lines represent 10 times the Hill radius. The times have been chosen to roughly correspond to the growth timescale for the isolation mass. The isolation mass is the mass that the protoplanet reaches at the end of oligarchic growth, when there are very few planetesimals left and the evolution enters the late stage.

Kokubo \& Ida (2002) derived the isolation mass of a powerlaw mass distribution

$$
\begin{aligned}
M_{\text {iso }}= & 0.16\left(\frac{\tilde{b}}{10}\right)^{3 / 2}\left(\frac{f_{\text {ice }} \Sigma_{1}}{10}\right)^{3 / 2} \\
& \times\left(\frac{a}{1 \mathrm{AU}}\right)^{(3 / 2)(2-\alpha)}\left(\frac{M_{*}}{M_{\odot}}\right)^{-1 / 2} M_{\oplus},
\end{aligned}
$$

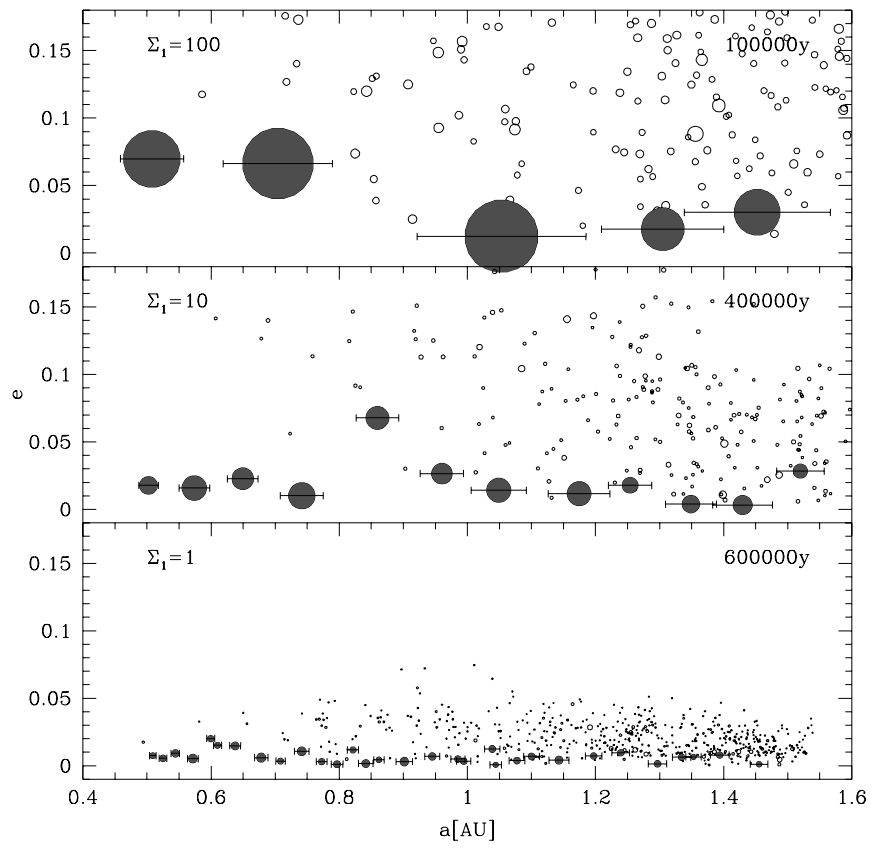

FIG. 8. -Eccentricity vs. semimajor axis for three different surface density distributions: $\Sigma_{1}=100,10$, and $1 \mathrm{~g} \mathrm{~cm}^{-2}$ (top to bottom). The runs shown here all have $\alpha=3 / 2$. The simulations are shown at $100,000,400,000$, and $600,000 \mathrm{yr}$, respectively (a few times the time required to grow isolation masses for the respective initial surface density). The filled circles represent those protoplanets that have grown 100 times the initial planetesimal mass $\left(1.5 \times 10^{25}, 1.5 \times 10^{24}\right.$, and $1.5 \times 10^{23} \mathrm{~g}$, respectively). [See the electronic edition of the Journal for a color version of this figure.]

where $\tilde{b}$ is the separation between protoplanets in units of $r_{\mathrm{H}}$ and $f_{\text {ice }}$ is the factor that the solid mass is increased because of the condensation of ice. In all simulations presented here, $f_{\text {ice }}=1$. The isolation mass between 0.5 and $1 \mathrm{AU}$ (assuming $\alpha=3 / 2$ ) ranges from $3 \times 10^{-3}$ to $6 \times 10^{-3}, 9.5 \times 10^{-2}$ to $2.17 \times 10^{-1}$, and 3.0 to $6.9 M_{\oplus}$ for $\Sigma_{1}=1,10$, and $100 \mathrm{~g} \mathrm{~cm}^{-2}$, respectively, for the three simulations.

The time required to grow a protoplanet of a given mass (Kokubo \& Ida 2002) is

$$
\begin{aligned}
t_{\text {grow }}= & 1.7 \times 10^{5} f^{-1}\left(\frac{\left\langle\tilde{e}^{2}\right\rangle^{1 / 2}}{6}\right)^{2}\left(\frac{M}{10^{26} \mathrm{~g}}\right)^{1 / 3} \\
& \times\left(\frac{f_{\text {ice }} \Sigma_{1}}{10}\right)^{-1}\left(\frac{a}{1 \mathrm{AU}}\right)^{\alpha+1 / 2}\left(\frac{M_{*}}{M_{\odot}}\right)^{-1 / 6} \mathrm{yr},
\end{aligned}
$$

where $f=6, f_{\text {ice }}=1$ at $1 \mathrm{AU}$ is the enhancement in mass due to condensation of volatiles, and $\left\langle\tilde{e}^{2}\right\rangle^{1 / 2} \equiv\left\langle e^{2}\right\rangle^{1 / 2} / h$ is the rms eccentricity in units of the reduced Hill radius of the protoplanet. Therefore, assuming that $\left\langle e^{2}\right\rangle^{1 / 2}=e_{\text {esc }}$ at $1 \mathrm{AU}$, it takes $\sim 2 \times$ $10^{4}, \sim 6 \times 10^{4}$, and $\sim 2 \times 10^{5}$ yr to grow a protoplanet with mass $M_{\text {iso }}$ for $\Sigma_{1}=100,10$, and 1, respectively. The protoplanets in Figure 8 are consistent with the equation (8), with masses of $3 \times 10^{-3}$ to $1.5 \times 10^{-2}, 6 \times 10^{-2}$ to $4 \times 10^{-1}$, and 1.6 to $7.8 M_{\oplus}$ for $\Sigma_{1}=1,10$, and $100 \mathrm{~g} \mathrm{~cm}^{-2}$, respectively.

Figure 9 shows protoplanet mass as a function of semimajor axis for three simulations with $\alpha=3 / 2$ and $\Sigma_{1}=100,10$, and $1 \mathrm{~g} \mathrm{~cm}^{-2}$. The circles, squares, and triangles represent the protoplanets in the $\Sigma_{1}=100,10$, and 1 simulations, respectively. The lines represent the isolation masses (eq. [7]) for each of the simulations. The solid line assumes a protoplanet separation of $10 r_{\mathrm{H}}$; the dashed line assumes $15 r_{\mathrm{H}}$. The simulations are consistent 


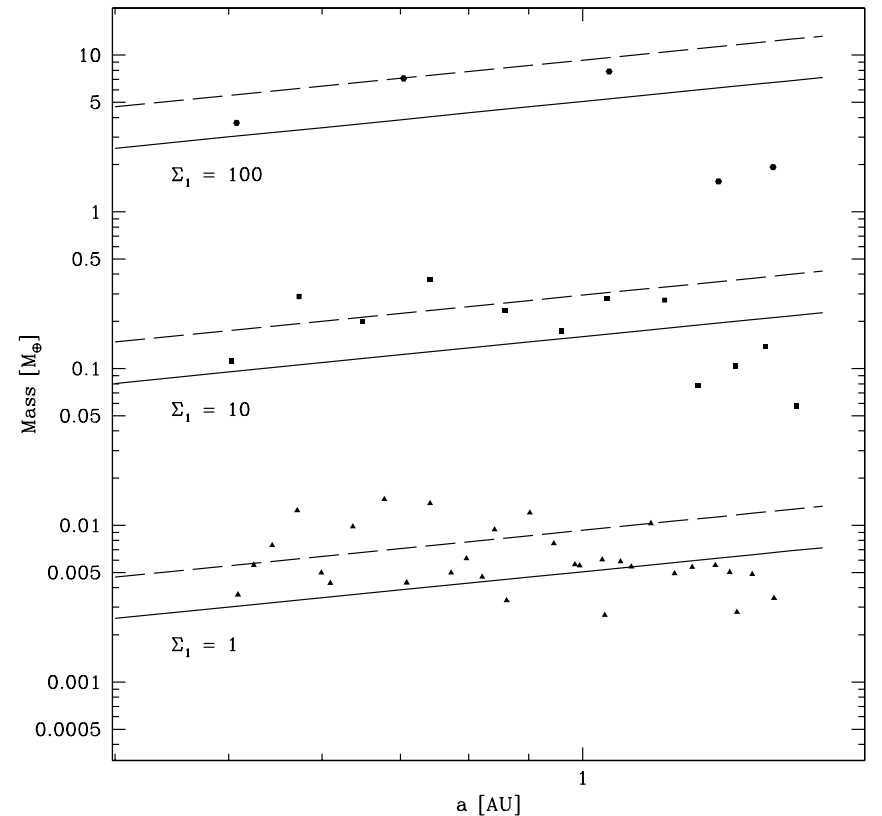

FIG. 9.- Protoplanet mass vs. semimajor axis for simulations of three different surface densities (as in Fig. 8). The circles represent protoplanets after $100,000 \mathrm{yr}$ with initial $\Sigma_{1}=100$, the squares protoplanets after $400,000 \mathrm{yr}$ with initial $\Sigma_{1}=10$, and the triangles protoplanets after $600,000 \mathrm{yr}$ with initial $\Sigma_{1}=1$. The lines show the theoretical isolation masses for these cases. The solid lines show the isolation masses assuming protoplanet separations of $10 r_{\mathrm{H}}$; the dashed lines show the isolation masses assuming separations of $15 r_{\mathrm{H}}$.

with the analytic predictions. The number of protoplanets decreases with increasing surface density, while the protoplanet masses increase with surface density.

We have also run simulations of various mass distributions. Figure 10 shows the results of three simulations with $\alpha=1 / 2$, $3 / 2$, and $5 / 2$ after $400,000 \mathrm{yr}$, keeping $\Sigma_{1}=10 \mathrm{~g} \mathrm{~cm}^{-2}$. The data points represent the protoplanets. The lines again represent the isolation masses as a function of semimajor axis for each distribution. Again the data are consistent with the theoretical predictions and with the results of Kokubo \& Ida (2002). Namely, the isolation mass increases with semimajor axis for $\alpha<2$ and decreases with semimajor axis for $\alpha>2$.

In summary, we have found that including fragmentation does affect the early evolution of protoplanets by altering the growth timescale. Our findings suggest that the collision model is important until large planetesimals/protoplanets emerge, at which point most collisions result in accretion events and the increase in velocity dispersion, eccentricity, and inclination of the background planetesimal population is dominated by the large bodies. The end results, however, are remarkably similar to those found using perfect merging.

\subsection{Collision Rates and Statistics}

Figure 11 shows the number of planetesimal collisions, the number of collisions that were interpolated, and the number of interpolated collisions that resulted in accretion or growth for all nine high-resolution simulations. Only $\sim 10 \%$ of collisions needed to be resolved using rubble piles. Almost all of the collisions that did not require full resolution resulted in growth. These general characteristics are independent of the initial conditions. The evolution of planetesimal growth, indicated by the shape of the collision curve, is slightly dependent on the initial surface mass density and the power law of the surface density distribution. The more massive the initial disk, the earlier growth starts

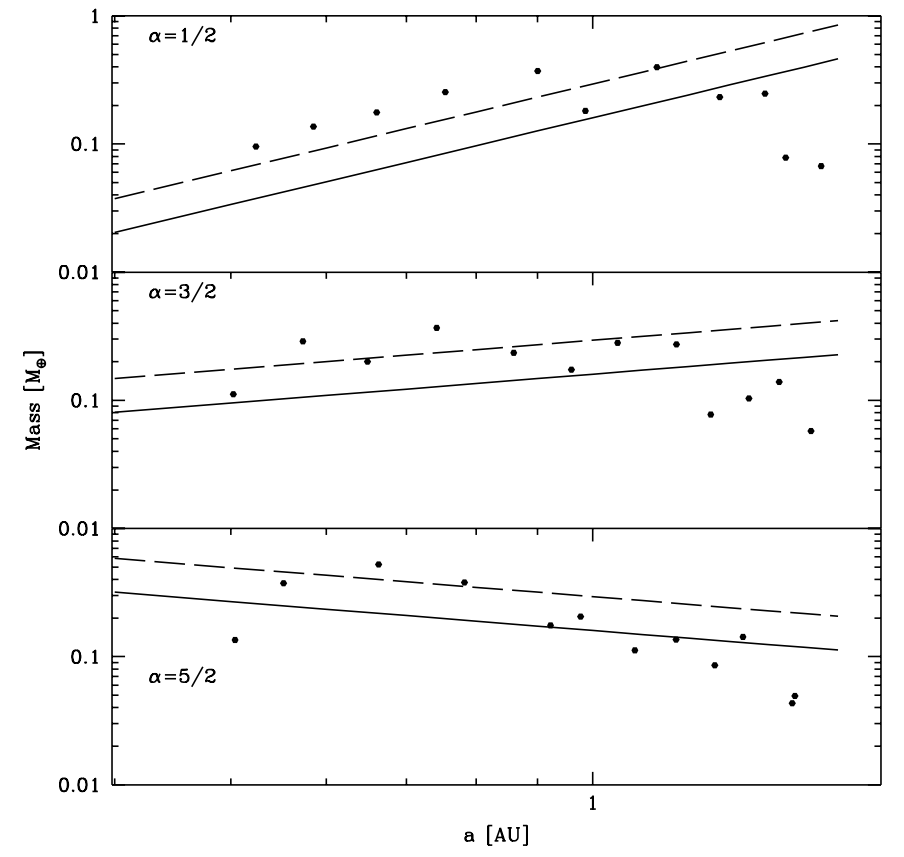

FIG. 10.-Protoplanet mass vs. semimajor axis for three different initial surface density distributions with power-law exponents $\alpha=1 / 2,3 / 2$, and $5 / 2$, respectively. $\Sigma_{1}=10 \mathrm{~g} \mathrm{~cm}^{-2}$ for the runs shown here. The lines represent the isolation masses for protoplanet separations of $10 r_{\mathrm{H}}$ (solid lines) and $15 r_{\mathrm{H}}$ (dashed lines). The protoplanets masses are in units of Earth's mass.

and the earlier runaway growth plateaus. Each disk initially has the same number of particles, so the more massive disks have larger particles with larger effective cross sections, and thus the collisional evolution is faster in these simulations.

Figure 12 shows the evolution of the collision parameters for the nine high-resolution cases. Figure $12 a$ shows the time

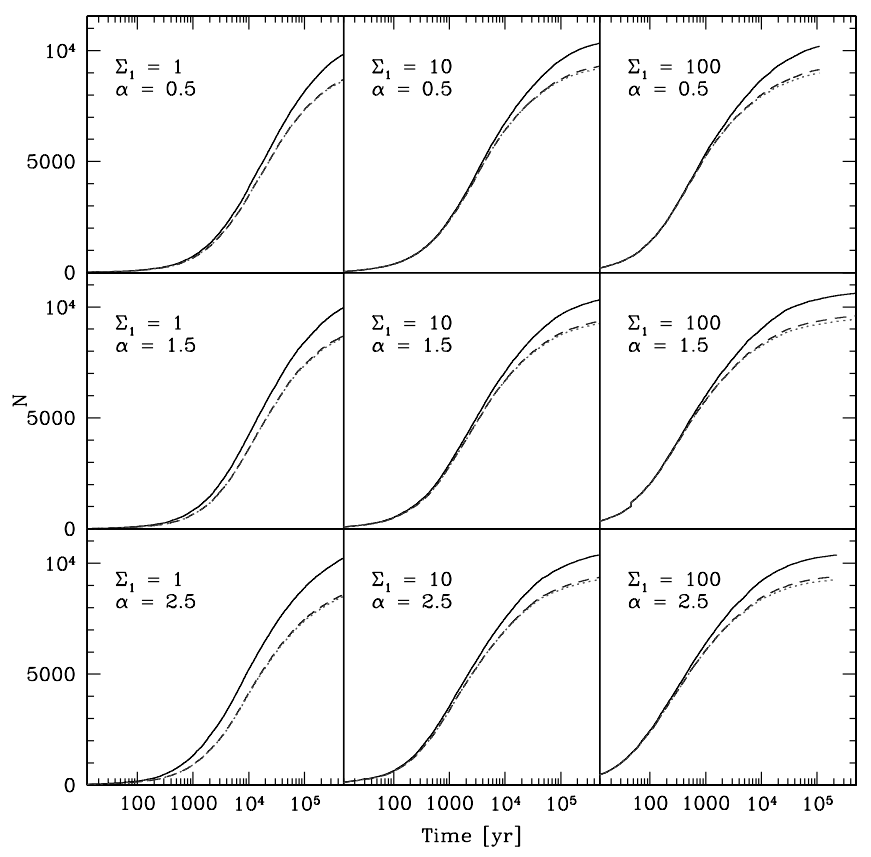

FIG. 11.-Cumulative plots of the number of collisions (solid lines), the number of interpolated collisions (dashed lines), and the number of interpolated collisions that resulted in accretion (dotted lines). An accretion event is a collision in which the mass of the largest postcollision remnant is larger than the mass of either colliding body. [See the electronic edition of the Journal for a color version of this figure.] 



FIG. 12.-(a) Average impact parameter (crosses) and mass ratio (dots) in logarithmic time bins. (b) Average impact speed for these collisions with the same binning. The error bars represent $50 \%$ of the most extreme values in that bin. $\Sigma_{1}$ and $\alpha$ are the same as in Fig. 11.

evolution of the average impact parameter and mass ratio. As a size distribution develops with the onset of runaway growth, the average impact mass ratio drops and the impact parameter remains roughly constant at $\sim 0.6$. The overall shape of the curves is similar for each run. Figure $12 b$ shows the evolution of impact speed. The average impact speed stays low throughout the simulation, which is consistent with the $\geq 90 \%$ accretion rate (Fig. 11). In most of the simulations the average impact speed grows as the planetesimals in the disk grow. This is due to gravitational scattering of planetesimals by the emerging protoplanets, increasing the eccentricities and the inclinations of the background planetesimals. The low-mass disk $\left(\Sigma_{1}=1\right.$, first column in Fig. $\left.12 b\right)$ shows a spike in impact speed starting at about $\sim 10^{3} \mathrm{yr}$. This is due to the initial excitement of background planetesimals when runaway growth begins in the innermost region of the disk, $\sim 0.5 \mathrm{AU}$. The increase in eccentricities of background planetesimals also occurs in the more massive disks, but the time resolution of the simulations is not fine enough to detect it in these faster evolving cases. Since planetesimal evolution takes the longest in $\Sigma_{1}=1$, the initial increase in impact speed at the beginning of runaway growth is detectable.

\subsection{Unresolved Debris}

As a result of our collision model, debris is created during most collisions. The debris is not followed directly $(\S 2)$. Instead, we keep track of only global properties. Figure 13 shows the evolution of the debris, along with that of the first, fifth, and tenth most massive protoplanets and the average-mass planetesimals, for comparison. ${ }^{4}$ All simulations were started with $1 \%$ of the total mass in planetesimals in unresolved debris. When the largest planetesimal (green line) reaches 50 to 100 times the initial mass of the planetesimals, the debris mass (black line) drops quickly.

\footnotetext{
4 The dust mass and mass of the planetesimals are output at a slightly different frequency in our simulations, which results in noise of order of a few in the debris located outside of the original disk bounds. This offset is responsible for the small dotted line spike in $\Sigma_{1}=100, \alpha=5 / 2$.
}

The spikes are due to individual collision events. By the end of the simulation the debris mass is at most an order of magnitude less than the initial condition, and in most cases the debris mass has dropped to zero. In most simulations there is a negligible amount of debris outside the initial protoplanetary disk (dotted line).

In almost all simulations the growth of the largest object went through two phases. In the first phase - runaway growth - the slope (growth rate) for the largest object in Figure 13 is close to 1. In all of the simulations except $\Sigma_{1}=1, \alpha=0.5$ this slope turns over and then drops below 1 (but remains positive). This turnover is an indication of oligarchic growth. The $\Sigma_{1}=1, \alpha=0.5$ simulation did not reach oligarchic growth. This conclusion is supported by Figures 14 and 15, which show snapshots of all high-resolution simulations at 500,000 yr (except for $\Sigma_{1}=100$, $\alpha=0.5$ shown at $110,000 \mathrm{yr}$ and $\Sigma_{1}=100, \alpha=2.5$ shown at $225,000 \mathrm{yr}$ ) in the $a-e$ and $a-m$ planes. Figure 14 shows that the isolation mass has been reached for all $\Sigma_{1}=10$ and $100 \mathrm{~g} \mathrm{~cm}^{-2}$ simulations because the protoplanets shown as filled circles are at least $10 r_{\mathrm{H}}$ from each other. Figure 15 shows that the $\Sigma_{1}$ simulations have just begun forming a small distinct population of massive objects, of which the $\Sigma_{1}=1, \alpha=0.5$ one is the most undeveloped.

For the simulation with $\Sigma_{1}=100$ there is a noticeable amount of mass outside the initial protoplanetary disk by $10,000 \mathrm{yr}$. This is because the protoplanets in these simulations are more massive and viscous stirring is more effective (i.e., $e$ and $i$ are higher for the planetesimals). As a result, some collisions between planetesimals occur outside the original protoplanetary disk. These collisions produce debris, but the debris in these outer regions is not swept up. Once the amount of mass in debris outside the initial protoplanetary disk increases, it cannot decrease. In these simulations it is considered "trash," and we keep track of it only to check mass conservation as a function of time. Regardless, as shown in Figure 16, by 500,000 yr the mass is always concentrated in a small number of massive protoplanets with a small amount of mass in planetesimals and a negligible amount of mass in debris for all runs. 


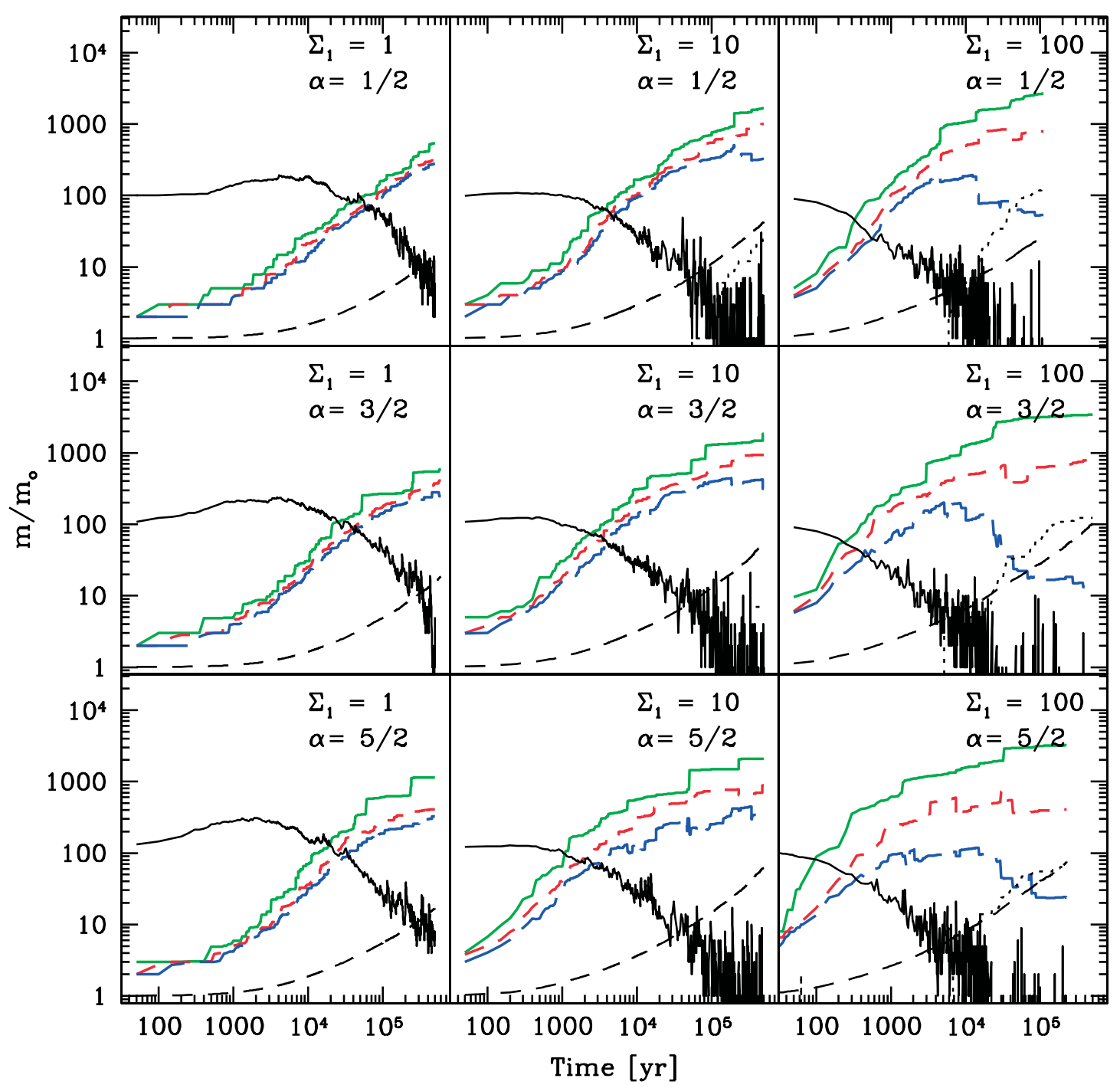

FIG. 13.-Evolution of the most massive planetesimals (solid green, dashed blue, and dashed red lines), the average planetesimals (dashed black line), the debris (solid black line), and the debris located outside the original disk bounds (dotted black line). The mass of the first, fifth, and tenth instantaneous largest planetesimals are shown in green, blue, and red, respectively. All are in units of the initial planetesimal mass $m_{0}$ for each simulation.

\subsection{Coefficient of Restitution}

It is unknown what material best describes planetesimals. In order to investigate the effect of planetesimal composition in a simple way, we conducted three sets of simulations using different normal coefficients of restitution $\left(\epsilon_{n}=0.1,0.5\right.$, and 0.8$)$. As a control we also ran one perfect merging simulation $\left(\epsilon_{n}=0\right)$ with the same initial conditions and no fragmentation. These simulations are lower resolution $\left(N=4000, m_{0}=3 \times 10^{23}\right)$, and the initial disk is significantly narrower $(\Delta a / a=0.085 \mathrm{AU}$ at $1 \mathrm{AU})$. As a result, planetesimals diffuse out of the initial annulus more quickly, so these simulations are run for a shorter period of time, $2 \times 10^{4} \mathrm{yr}$.

Figure 17 shows the mass versus time in the top panels and velocity dispersion versus time in the bottom panels for these cases. The solid line in the top panels shows the maximum instantaneous mass, and the dashed line shows the average mass. The coefficient of restitution appears to have less of an effect on the growth of the planetesimals than including a fragmentation model. The simulations with $\epsilon_{n}>0$ have average planetesimal masses that are indistinguishable from each other, and the range in maximum mass is also similar between cases. The average mass, maximum mass, and velocity dispersion of the $\epsilon_{n}=0$ case are slightly lower than for the other simulations. However, the $\epsilon_{n}>0$ simulations do show significant spread in outcome based on random changes in the initial conditions.

The bottom panels in Figure 17 show the velocity dispersion both weighted by mass (dashed line) and unweighted (solid line). The unweighted velocity dispersion is given by

$$
\sigma=\sqrt{\frac{\sum_{i=1}^{N}\left|\boldsymbol{v}_{i}-\boldsymbol{v}_{k i}\right|^{2}}{N-1}},
$$

where $\boldsymbol{v}_{i}$ is the instantaneous velocity of particle $i, \boldsymbol{v}_{k i}$ is the Keplerian velocity at the instantaneous position of particle $i$, and $N$ is the instantaneous number of particles. The unweighted velocity dispersion follows the velocity dispersion of the most numerous particles, which in this case are the background planetesimals. The mass-weighted velocity dispersion is given by

$$
\sigma_{v m}=\sqrt{\frac{\sum_{i=1}^{N} m_{i}\left|\boldsymbol{v}_{i}-\boldsymbol{v}_{k i}\right|^{2}}{\sum_{i=1}^{N} m_{i}}},
$$




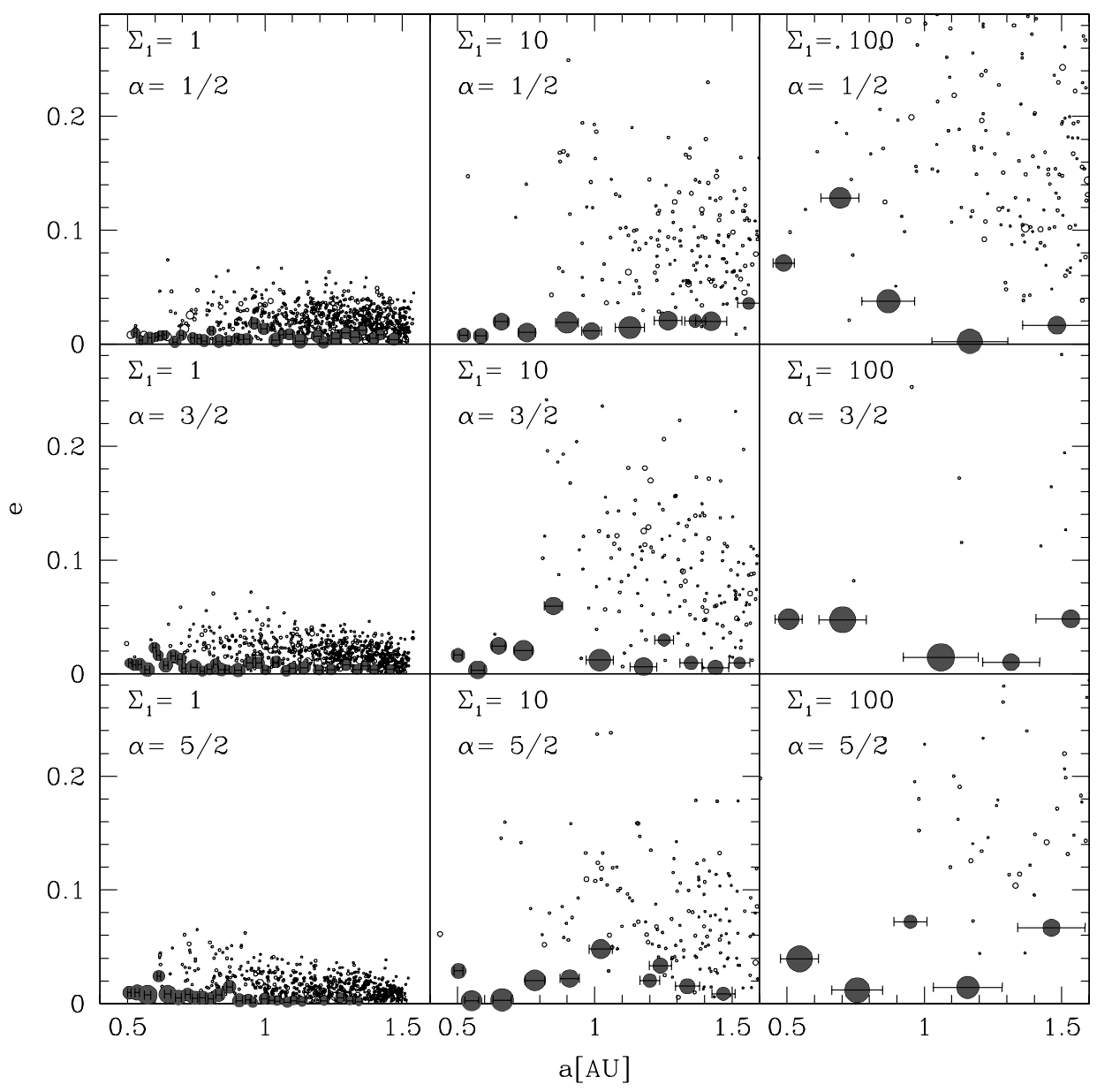

Fig. 14. - Particle locations in semimajor axis-eccentricity space for all high-resolution simulations. All simulations are shown at 500,000 yr except $\Sigma_{1}=100$, $\alpha=1 / 2$, which is shown at $110,000 \mathrm{yr}$, and $\Sigma_{1}=100, \alpha=5 / 2$, which is shown at 225,000 yr. As in Figs. 5 and 8 , the filled circles show protoplanets and the horizontal error bars represent $10 r_{\mathrm{H}}$. [See the electronic edition of the Journal for a color version of this figure.]

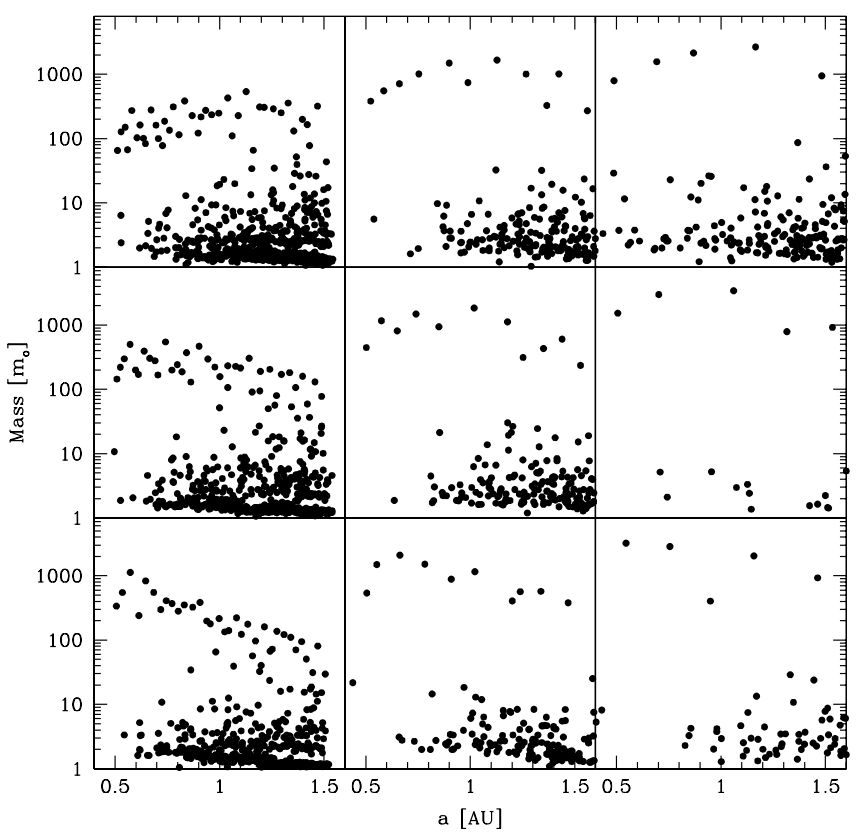

FIG. 15.-Mass of the planetesimals in units of $m_{0}$ shown in Fig. 14.

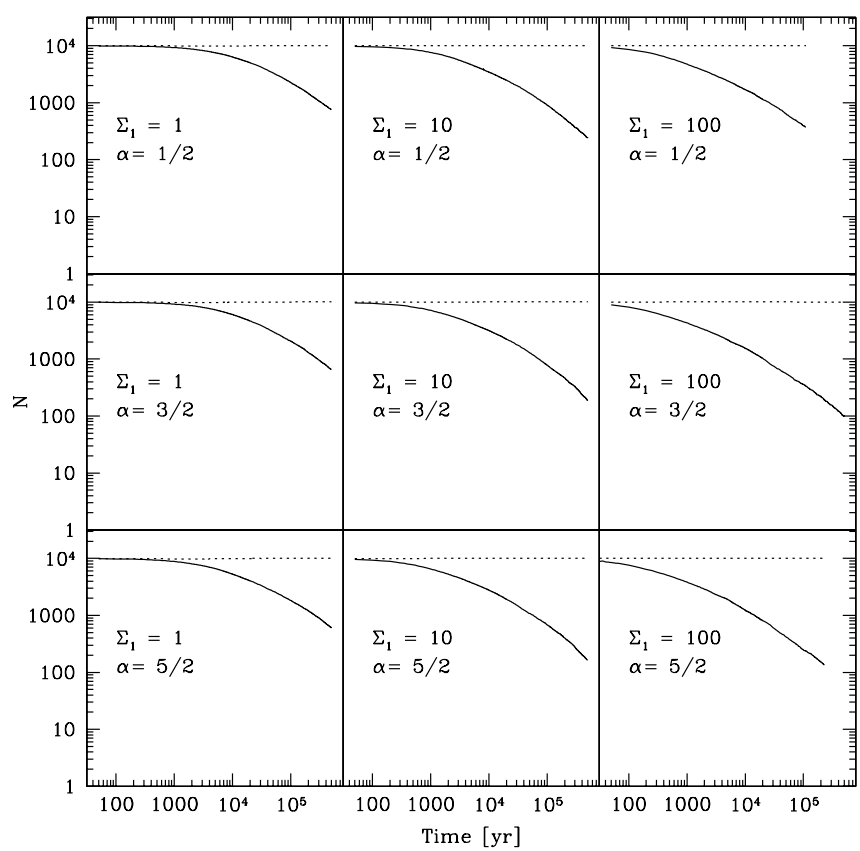

FIG. 16.-Evolution of the number of particles (i.e., both planetesimals and protoplanets; solid line) and total mass in planetesimals and protoplanets in units of the initial mass (dotted line). 

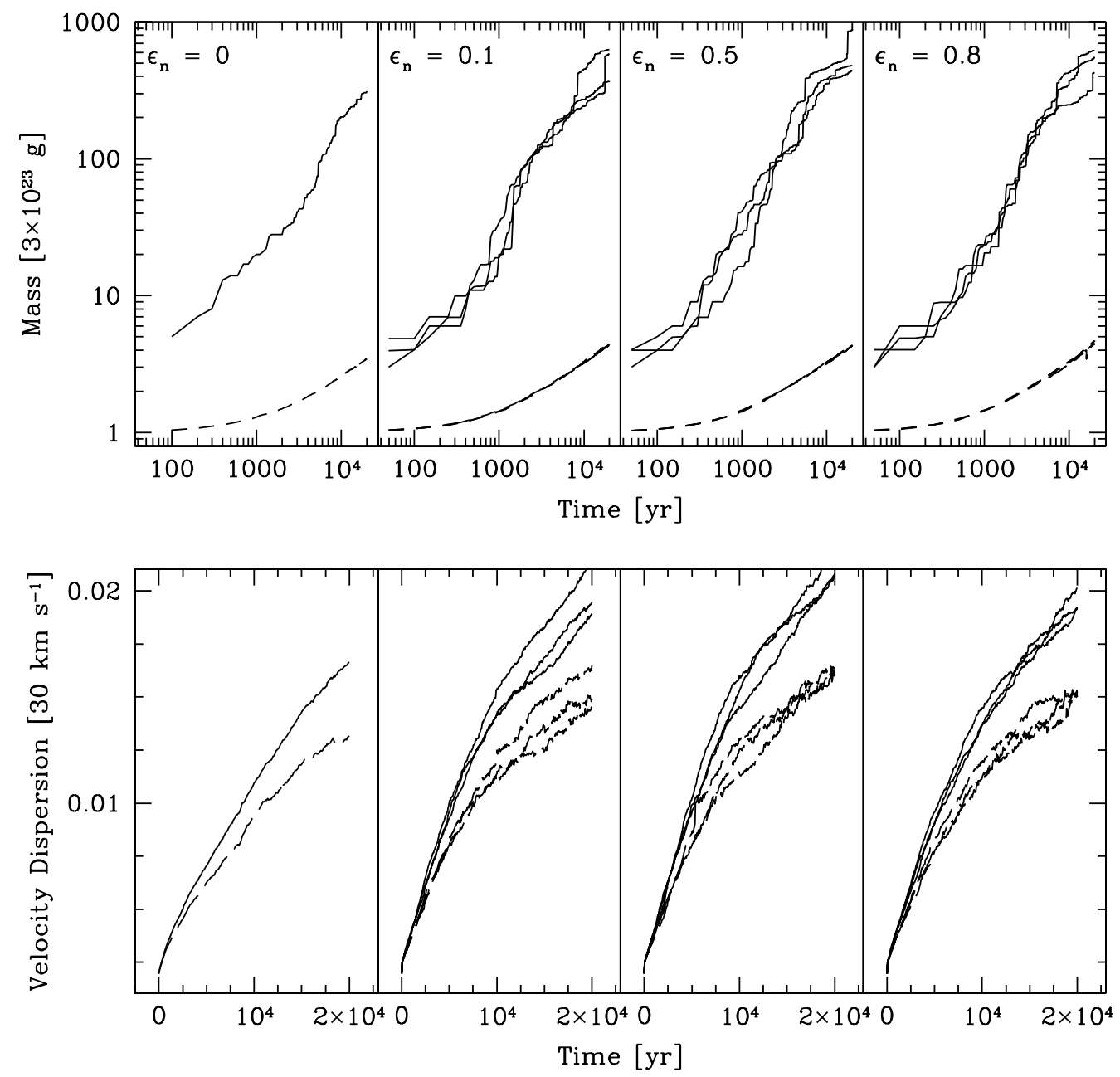

FIG. 17.-Mass as a function of time (top) and velocity dispersion as a function of time (bottom) for $\epsilon_{n}=0$ (perfect merging), $0.1,0.5$, and 0.8 . For each $\epsilon_{n}>0$, three simulations were conducted. Each is represented by a separate line in the plots. The solid lines in the top panels are for the largest instantaneous mass. The dashed line shows the average mass. In the bottom panels the solid line shows the velocity dispersion and the dashed line shows the velocity dispersion weighted by mass.

where $m_{i}$ is the mass of particle $i$. This quantity is dominated by the velocity dispersion of the more massive planetesimals. As a result, $\sigma_{v m}$ is less than $\sigma$ and the difference between them grows as the largest planetesimals grow. The velocity dispersions also show little dependence on $\epsilon_{n}$.

The energy change in the center-of-mass frame of a system of two smooth, colliding spheres is given by (Araki \& Tremaine 1986)

$$
\Delta E=-\frac{1}{2} \mu_{r}\left(1-\epsilon_{n}^{2}\right) v_{n}^{2}
$$

where $\mu_{r}$ is the reduced mass and $v_{n}$ is the normal component of relative impact velocity. We have shown in past work that this relationship holds for rubble-pile collisions (Leinhardt et al. 2000). Thus, the lack of dependence of largest mass and velocity dispersion on coefficient of restitution suggests that collisions, although the primary growth mechanism, do not dominate the velocity field during most of protoplanetary growth. The effects of planetesimal collisions could be important in the early stage of terrestrial planet formation before the emergence of large planetesimals and protoplanets. Viscous stirring by the protoplanets becomes dominant over any change in the velocity field due to a collision by $10^{4} \mathrm{yr}$. As a result, we conclude that fragmentation is also not particularly important during most of the runaway growth and beyond.

\section{CONCLUSIONS}

We have completed a series of high-resolution direct $N$-body simulations of terrestrial planet formation. We have included a self-consistent planetesimal collision model in which gravity is the dominant mechanism for determining the collision outcome. We have determined that fragmentation is unimportant in determining the final outcome of protoplanet formation in a gas-free environment. The fragmentation model that we employed did affect the rate of planetesimal evolution, suggesting that fragmentation could be important in the early phase of runaway growth, but the end result, after oligarchic growth, was consistent with perfect merging simulations. We have also found that the coefficient of restitution does not affect the growth of planetesimals over a timescale of $10^{4} \mathrm{yr}$. The largest planetesimals dominate the growth through viscous stirring; the material properties are unimportant.

\section{FUTURE WORK}

It is possible that fragmentation could change the surface density distribution in a gaseous disk. Smaller fragments could migrate 
radially within the disk and may flatten or steepen the mass distribution and thus change the number, location, and mass of the protoplanets. We will investigate this in future work. We also did not fully investigate the debris initial condition. If the mass of debris is of the same order as the larger planetesimals, the debris could have a significant dynamical affect on the larger planetesimals. Although this situation did not develop in any of the environments that we investigated, we always started with a debris population that was $1 \%$ the mass of the larger planetesimals. There may be some critical initial mass that is required to cause a noticeable dynamical affect. In order to study this in detail we would also need to include the effect of dynamical friction of the debris component on the planetesimals and gravitational focusing of the debris by the large planetesimals. In the simulations presented here we neglected gravitational focusing of the debris component because we made simplifying assumptions about the mass distribution and orbits of the debris: the debris was distributed smoothly though out the annulus and all debris was assumed to be on circular orbits. Adding gravita- tional focusing would not make the result more accurate in light of the above assumptions. In addition, there was never enough debris to significantly change the growth evolution of the protoplanets. In the next set of simulations, in which the mass of the initial debris component will be increased by orders of magnitude, gravitational focusing may become an important growth mechanism and must be investigated. We would also like to complete a simulation without (i.e., $f=1$ ) the expansion parameter to determine a true terrestrial planet formation timescale and to determine the distribution and evolution of spin states.

This material is based on work supported by NASA under grants NGT550454 and NAG511722 issued through OSS. The authors would like to thank E. Kokubo for a careful and thoughtful review of this paper. Z. M. L. would also like to thank KITP at UCSB, where a significant amount of work for this paper was completed.
Agnor, C. B., \& Ward, W. R. 2002, ApJ, 567, 579

Araki, S., \& Tremaine, S. 1986, Icarus, 65, 83

Asphaug, E., \& Benz, W. 1996, Icarus, 121, 225

Asphaug, E., Ostro, S. J., Hudson, R. S., Scheeres, D. J., \& Benz, W. 1998, Nature, 393, 437

Asphaug, E., Ryan, E. V., \& Zuber, M. T. 2002, in Asteroids III, ed. W. F. Bottke, A. Cellino, P. Paolicchi, \& R. P. Binzel (Tucson: Univ. Arizona Press), 463

Beaugé, C., \& Aarseth, S. J. 1990, MNRAS, 245, 30

Greenberg, R., Hartmann, W. K., Chapman, C. R., \& Wacker, J. F. 1978, Icarus, 35,1

Holsapple, K. A. 1994, Planet. Space Sci., 42, 1067

Kokubo, E., \& Ida, S. 1995, Icarus, 114, 247 1996, Icarus, 123, 180

1998, Icarus, 131, 171

2000, Icarus, 143, 15

2002, ApJ, 581, 666

Lecar, M., \& Aarseth, S. J. 1986, ApJ, 305, 564

Leinhardt, Z. M., \& Richardson, D. C. 2002, Icarus, 159, 306

Leinhardt, Z. M., Richardson, D. C., \& Quinn, T. 2000, Icarus, 146, 133

\section{REFERENCES}

Lissauer, J. J. 1993, ARA\&A, 31, 129

Love, S. G., \& Ahrens, T. J. 1996, Icarus, 124, 141

Makino, J., Fukushige, T., Funato, Y., \& Kokubo, E. 1998, NewA, 3, 411

Michel, P., Benz, W., Tanga, P., \& Richardson, D. C. 2001, Science, 294, 1696

Pravec, P., Harris, A. W., \& Michalowski, T. 2002, in Asteroids III, ed. W. F.

Bottke, A. Cellino, P. Paolicchi, \& R. P. Binzel (Tucson: Univ. Arizona Press), 113

Richardson, D. C., Elankumaran, P., \& Sanderson, R. E. 2005, Icarus, 173, 349 Richardson, D. C., Leinhardt, Z. M., Melosh, H. J., Bottke, W. F., \& Asphaug, E. 2002, in Asteroids III, ed. W. F. Bottke, A. Cellino, P. Paolicchi, \& R. P. Binzel (Tucson: Univ. Arizona Press), 501

Richardson, D. C., Quinn, T., Stadel, J., \& Lake, G. 2000, Icarus, 143, 45

Ryan, E. V., Hartmann, W. K., \& Davis, D. R. 1991, Icarus, 94, 283

Safronov, V. S. 1969, Evoliutsiia Doplanetnogo Oblaka i Obrazovanie Zemli i

Planet (Moscow: Nauka) (English transl. Evolution of the Protoplanetary

Cloud and Formation of Earth and the Planets NASA Tech. Transl. F-677;

Jerusalem: Israel Program Sci. Transl., 1972)

Stadel, J. G. 2001, Ph.D. thesis, Univ. Washington

Wetherill, G. W., \& Stewart, G. R. 1989, Icarus, 77, 330

-. 1993, Icarus, 106, 190 